\title{
Stroma-shaped pancreatic intratumoural tissue heterogeneity and architecture linked to clinical outcomes
}

With this
approach,
eight types of
PDAC tumour
gland were
identified

In a single-cell RNA sequencing (scRNA-seq) study published in Cell, a team of researchers have identified cancer cell phenotypes that can be used to classify tumour glands from patients with pancreatic ductal adenocarcinoma (PDAC). This typing of tumour glands was found to correlate with patient survival and response to therapy.

The tumour stroma of PDAC is mainly composed of cancer-associated fibroblasts (CAFs). However, examining the role of CAFs in PDAC biology has led to contradictory findings that show they can either suppress or enhance PDAC growth and the potential for metastatic dissemination, suggesting a complex relationship between PDAC tumour cells and CAFs.

"Our prior work characterizing PDAC heterogeneity using scRNA-seq with circulating tumour cells (CTCs) and primary tumours of the genetically engineered KPC mouse model was the initial driving force of this project," explains corresponding author David Ting. This work revealed expression profiles among CTCs that were either proliferative (PRO) or indicative of epithelial-to-mesenchymal transition

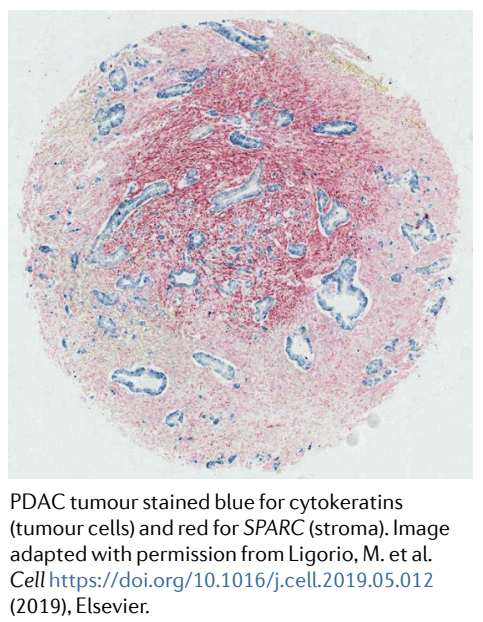

(EMT) and metastasis. "Interestingly, CTC-enriched genes were found to be elevated in a unique population of primary tumour cells at the tumour-stromal interface," says Ting. "This suggested that tumour cell interactions with stromal CAFs might be important in intratumoural cellular heterogeneity."

To further probe the causes of heterogeneity in PDAC and the role of CAFs, the investigators expanded upon previous scRNA-seq studies to provide functional characterization of the heterotypic responses of PDAC cells to CAFs.

"We used a well-controlled co-culture system of human PDAC and CAFs to understand the impact of different PDAC:CAF ratios on the generation of single-cell heterogeneity," explains Ting. After performing scRNA-seq on these cells, gene set enrichment analyses showed grouping into either PRO or EMT classes, supporting earlier work by the authors. They also identified a novel, aggressive double-positive phenotype with both PRO and EMT features that was associated with the highest CAF:PDAC ratio, as well as a double-negative phenotype associated with the absence of CAFs. These findings demonstrated the importance of PDAC-CAF crosstalk in PDAC cell heterogeneity.

Using patient-derived PDAC cell lines, the contribution of CAF-secreted factors using both mass cytometry (CyTOF) and flow cytometry was examined to evaluate protein markers of the PRO and EMT phenotypes. In the presence of CAF-derived conditioned media, a shift towards PRO, EMT and the double-positive phenotypes was seen, confirming the scRNA-seq findings at the protein level. "Using the same cell lines, we then demonstrated functional differences in both in vitro and in vivo model systems, which provided a comprehensive validation of the single-cell phenotypes identified by scRNA-seq," reports Ting.

"Lastly, using RNA in situ hybridization, we stained a large cohort $(n=220)$ of human PDAC primary tumours that were untreated or FOLFIRINOX-treated, and then analysed $>300,000$ single cells with high-content image analysis," reports Ting. This analysis enabled evaluation of single-cell heterogeneity within a spatial, architectural context, as well as the assessment of multiple glands within a tumour. With this approach, eight types of PDAC tumour gland were identified, each composed of cells with different markers of the PRO and EMT phenotypes. "Furthermore, we noted that the composition of each of these cell types within discrete tumour glands correlates with stroma content, patient survival, and response to preoperative FOLFIRINOX chemotherapy, which is not seen with single-cell analysis alone," says Ting.

"This study has demonstrated that PDAC cells are grouped within different gland units within a single tumour and that these gland units can vary based on regional differences in stromal content," concludes Ting. "This may explain the intrinsic and adaptive resistance of PDAC to a wide variety of therapies compared with other cancers." The team now plan to use their models to evaluate new therapeutic strategies to combat PDAC intratumoural gland heterogeneity and provide rationale for future human clinical trials.

Iain Dickson

ORIGINAL ARTICLE Ligorio, M. et al. Stromal microenvironment shapes the intratumoral architecture of pancreatic cancer. Cell https:// doi.org/10.1016/j.cell.2019.05.012 (2019) 